\title{
Residential greenness: current perspectives on its impact on maternal health and pregnancy outcomes
}

\author{
Rachel F Banay',* \\ Carla P Bezold ${ }^{2}, *$ \\ Peter James ${ }^{1-3}$ \\ Jaime E Hart ${ }^{1,3}$ \\ Francine Laden ${ }^{1-3}$ \\ 'Department of Environmental \\ Health, ${ }^{2}$ Department of Epidemiology, \\ Harvard T.H. Chan School of Public \\ Health, ${ }^{3}$ Channing Division of \\ Network Medicine, Department of \\ Medicine, Brigham and Women's \\ Hospital, Harvard Medical School, \\ Boston, MA, USA \\ *These authors contributed equally \\ to this work
}

This article was published in the following Dove Press journal:

International Journal of Women's Health

28 February 2017

Number of times this article has been viewed

\begin{abstract}
Recent research in environmental epidemiology has attempted to estimate the effects of exposure to nature, often operationalized as vegetation, on health. Although many analyses have focused on vegetation or greenness with regard to physical activity and weight status, an incipient area of interest concerns maternal health and birth outcomes. This paper reviews 14 studies that examined the association between greenness and maternal or infant health. Most studies were cross-sectional and conducted in birth cohorts. Several studies found evidence for positive associations between greenness and birth weight and maternal peripartum depression. Few studies found evidence for an association between greenness and gestational age or other birth outcomes, or between greenness and preeclampsia or gestational diabetes. Several assessed effect modification by individual or area-level socioeconomic status and found that effects were stronger among those of lower socioeconomic status. Few studies conducted mediation analyses of any kind. Future research should include more diverse birth outcomes and focus on maternal health (especially mental health) and capitalize on richer exposure information during pregnancy rather than cross-sectional assessment at birth.
\end{abstract}

Keywords: greenness, green space, birth outcomes, prenatal health, infant health

\section{Introduction}

In recent years, environmental epidemiologists have turned their attention to the potential health effects of exposure to nature. Exposure to nature is thought to affect health in several ways. Natural environments may promote health by providing opportunities for routine and recreational physical activity. ${ }^{1}$ They may have a therapeutic effect on mental health. ${ }^{2}$ They may facilitate social interactions and social cohesion, demonstrated to provide benefits to health. ${ }^{3}$ Vegetation may also lower harmful exposures, for example, by filtering air pollution, ${ }^{4}$ buffering noise, ${ }^{5}$ or alleviating thermal discomfort. ${ }^{6}$ In concert, these effects could help produce healthier pregnancies and better birth outcomes.

In scientific analyses, exposure to nature is often conceptualized as exposure to vegetation, hence the development of a literature concerned with greenness or green space. So far, studies have provided some evidence for a positive association between greenness exposure and physical activity, weight, heart disease, mental health, and developmental outcomes, among others. ${ }^{2}$ Some of these topics have received greater attention than others, and the evidence on a relationship between greenness and maternal health and birth outcomes is still developing. Researchers continue to attempt to isolate the effects of nature from socioeconomic status and to disentangle it from
Correspondence: Carla P Bezold

Department of Epidemiology, Harvard

T.H. Chan School of Public Health,

Landmark Center, 3 West, 40 I

Park Drive, Boston, MA 02215, USA

Tel + I $617525229 \mid$

Fax +I 6175252578

Email cpbI88@mail.harvard.edu
International Journal of Women's Health 2017:9 |33-|44

(c) (1) (\$) ๑ 2017 Banay et al. This work is published and licensed by Dove Medical Press Limited. The full terms of this license are available at https://www.dovepress.com/terms.php cc. hereby accept the Terms. Non-commercial uses of the work are permitted without any further permission from Dove Medical Press Limited, provided the work is properly attributed. For permision for commercial use of this work, please see paragraphs 4.2 and 5 of our Terms (https://www.doveperess. com/terms.php). 
other constructs related to the contextual environment, such as walkability, perceived safety, access to destinations, and more. This paper reviews the evidence to date on greenness and maternal health and birth outcomes and suggests future research directions.

\section{Methods}

MEDLINE (PubMed) and Embase were searched for relevant studies, with the final search for inclusion conducted on September 30, 2016. Titles and abstracts were searched for two groups of terms: 1) exposure: "greenness" or "green space" and 2) outcome: "birth outcomes", "preterm birth", "premature birth", "low birth weight (LBW)", "intrauterine growth retardation", "small for gestational age (SGA)", "pregnancy outcomes", "maternal outcomes", "reproductive outcomes", "preeclampsia", "gestational diabetes", "spontaneous abortion", "pregnancy loss", "maternal depression", "postpartum depression", or "peripartum depression". The search was further limited to studies in humans and those available in English.

Identified titles and abstracts were screened for relevance. The study included articles that presented original research including primary data analysis such as cross-sectional, cohort, or ecological studies and reported at least one measure of association for exposure to greenness or green space and one or more of the outcomes of interest. Articles without original data, such as reviews or commentaries, articles that presented greenness as part of a composite exposure rather than independently, and articles that did not report on outcomes of interest were excluded. All articles were screened by one reviewer and any questions about article inclusion were resolved through discussion among the authors. In addition, this study reviewed the reference lists of included reviews and other relevant publications for studies that were not identified in the searches. After the initial screening of titles and abstracts full texts were further reviewed for relevance, applying the same inclusion criteria. Data were extracted on the first author and publication year, study population (including country, population size, and year[s] of enrollment), study design, exposure, outcome(s), whether the study assessed effect measure modification or mediation, and the main findings. All articles were abstracted by one reviewer and checked by another.

\section{Results}

After duplicates were removed, the initial search yielded 16 articles. Of these, three were excluded - two based on study design (one review and one commentary) and one for lack of a relevant outcome. One study was further excluded while reviewing full texts for lacking an exposure measure of interest, and two additional articles were identified from publication reference lists. Details of the search are presented in Figure 1.

Ultimately 14 relevant articles were identified. Table 1 presents an overview of the included studies. The studies on this topic dealt with a defined set of outcomes, including birth weight, gestational age, preterm birth, head circumference and infant mortality, and among mothers, gestational diabetes, preeclampsia, and peripartum depression. Most studies took advantage of birth cohort data in analyzing associations between maternal address and maternal and infant health; however, most of these studies were cross-sectional, defining exposure based on residence at the time of birth, and were unable to explore how long the exposure had been present, if at all, before the outcome occurred. Many studies, but not all, were able to adjust for maternal age, smoking and alcohol use, and individual or area socioeconomic status.

\section{Exposure metrics}

To assess greenness or green space exposure, researchers generally used one or both of two strategies. The first, the predominant greenness exposure metric, involved characterizing vegetative density around participants' home addresses or within an administrative unit (eg, Census tract) using remotely sensed greenness information. These data came from vegetation indices, predominantly the Normalized Difference Vegetation Index (NDVI). Such vegetation indices, derived from satellite imagery, measure the light reflected and absorbed from the earth's surface during photosynthesis, arriving at an estimate of vegetative density. This information can be used to characterize greenness at varying spatial and temporal scales. In the studies that were reviewed, researchers used different spatial contexts to evaluate greenness; for example, some studies analyzed residential greenness by computing vegetation levels within buffers around a geocoded residential address (between 50 and 1,250 m), whereas others analyzed vegetation levels within an administrative unit. In addition, studies varied as to the timing of exposure during which they characterized greenness (at a single moment or averaging over a specific duration).

The other approach researchers took to characterizing greenness, less commonly used or used in tandem with the NDVI metric, was to quantify "green" land uses within a certain proximity to the residence or within a given administrative unit. This technique was applied differently depending on data sources available, but typically involved analyzing 


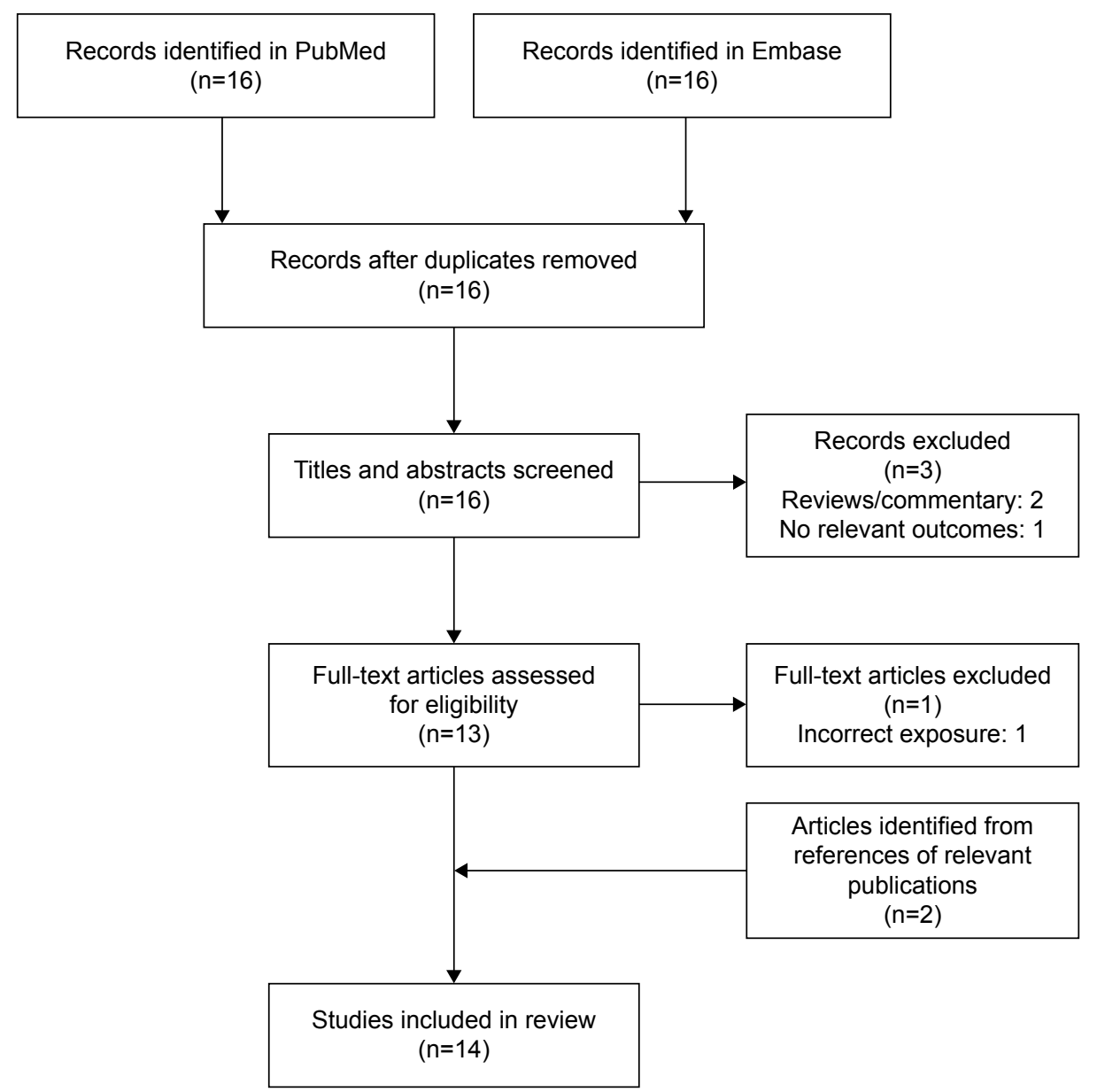

Figure I Results of search for original articles with results for greenness and maternal health and pregnancy outcomes.

local land use files, which classify land parcels according to their predominant use. Qualifying land uses included parks, forests, recreation areas, sports fields, golf courses, etc; researchers assigned exposure values based on the proximity of such green land uses to the residence, or the proportion of green land uses within an administrative boundary or defined buffer distance around the residence. Some studies considered both vegetation index and land use approaches.

Vegetation indices such as NDVI are available at varying spatial and temporal resolutions. The most commonly accessed sources provide a spatial resolution of 30-250 m every 16 days. Within each study, authors generally used data from a single time point that captured the maximum variability in greenness for their population of interest. The time points chosen for each study therefore varied depending on the climate and geography where study participants lived, but all studies that took this approach relied on values measured between June and October. One study, by Casey et al, assigned each subject the average NDVI value of the three seasons prior to the child's birth. ${ }^{7}$ In contrast to studies that relied on a single measure of peak greenness, this approach to exposure characterization allowed for the incorporation of seasonal changes in greenness.

To assign individual exposures, studies most commonly assigned the average value of the vegetation index within one or more buffer sizes around a participant's home. The varying buffer sizes used to characterize greenness captured different neighborhood definitions. For example, a smaller buffer size may capture the environment immediately visible outside a person's home, while a larger buffer size may capture an average walkable area. ${ }^{2}$ Many studies considered a range of buffer sizes in their analyses.

A question that has persisted in this research area concerns whether greenness metrics capture the quality of a given area, for example, whether a green space is safe, clean, pleasant, etc, or whether participants make use of it or not. The papers summarized in this review did not particularly address these concerns by incorporating environmental quality (eg, through environmental assessments) or participant use of green spaces (eg, through surveys). 


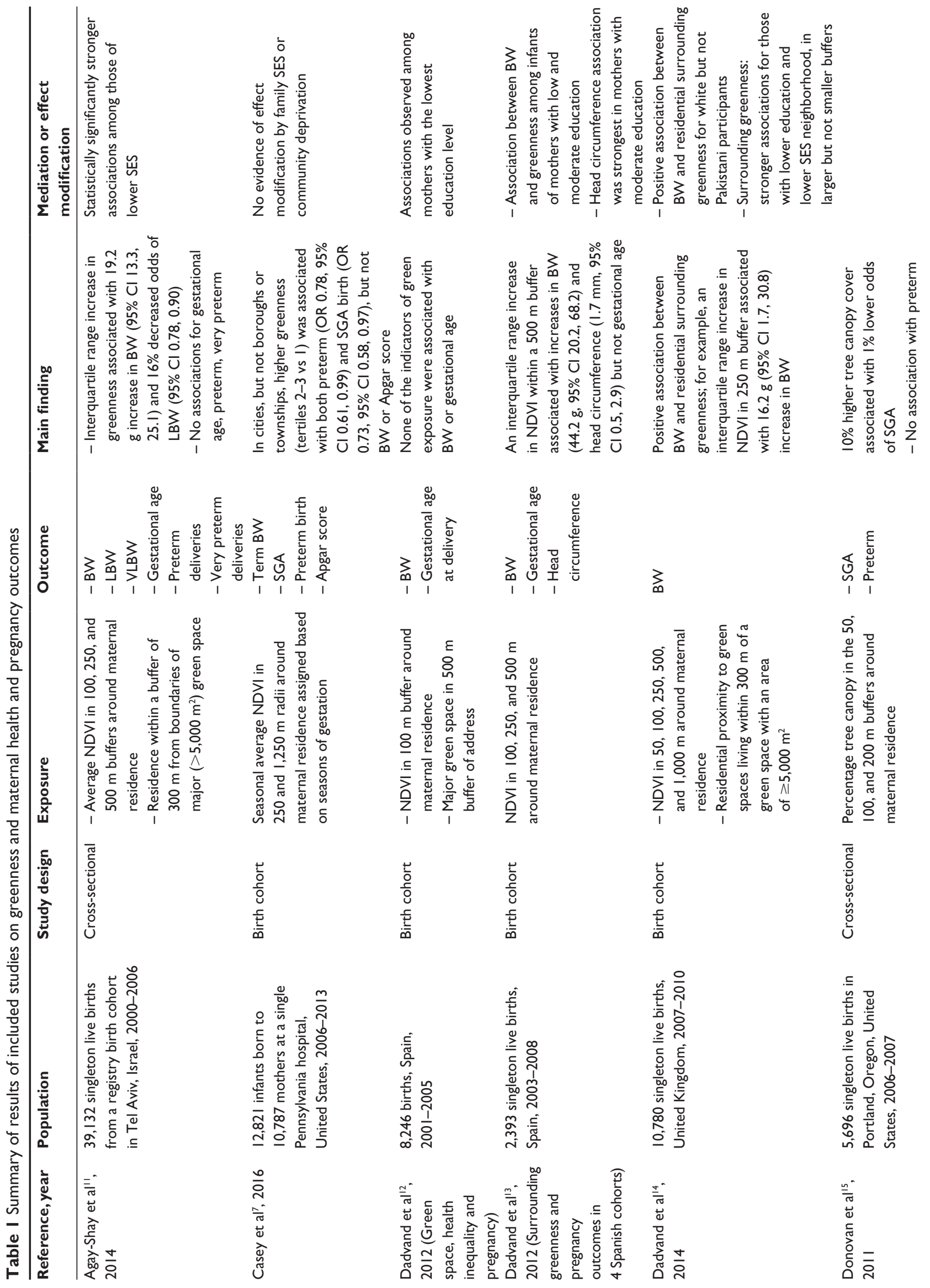



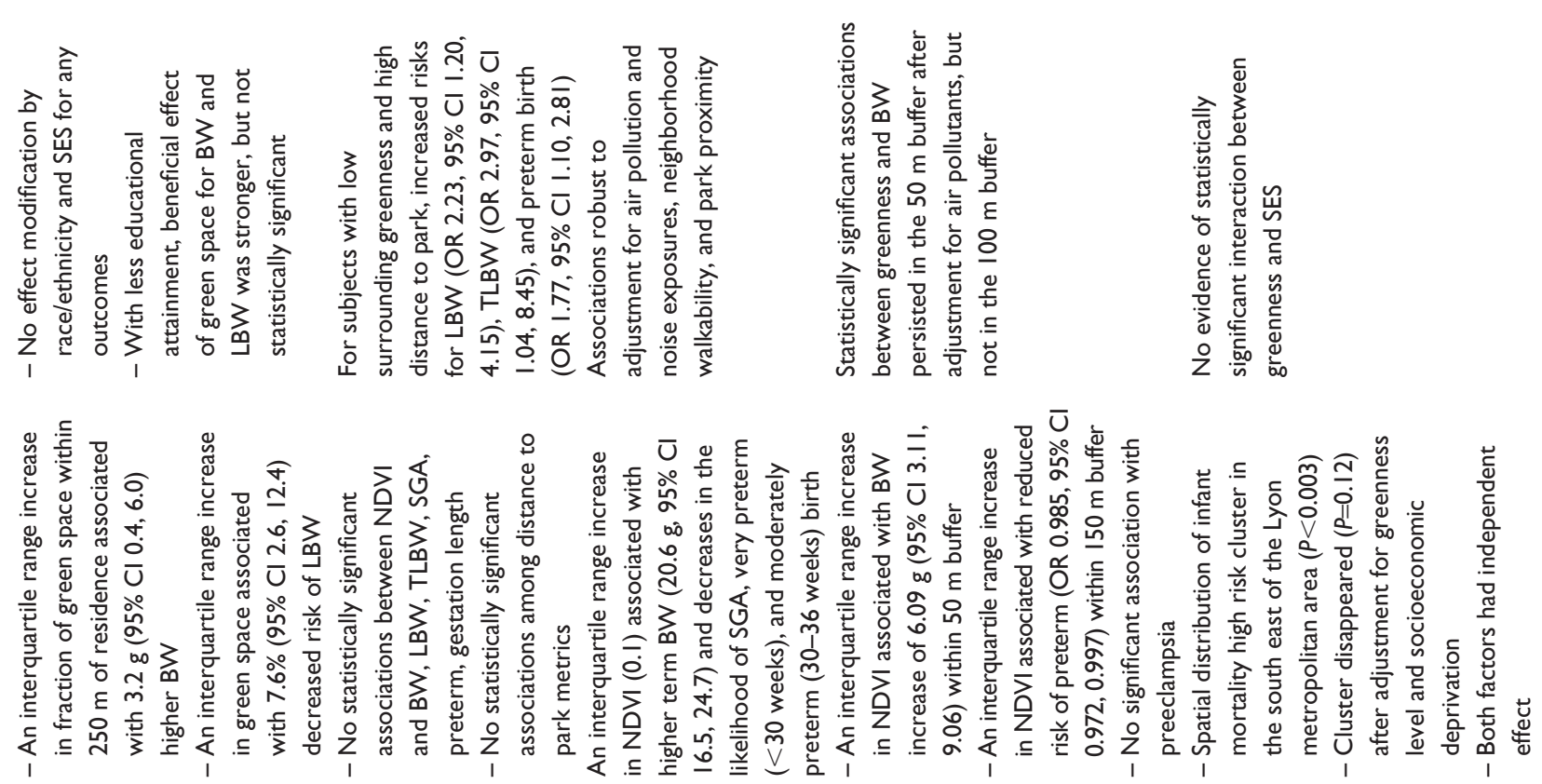

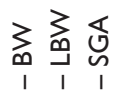

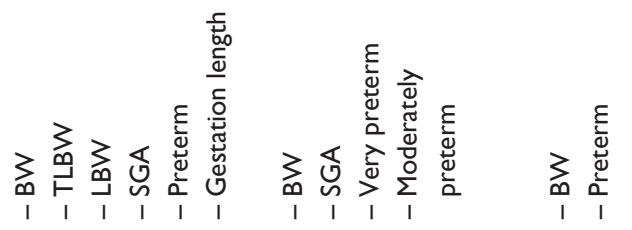

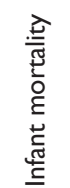
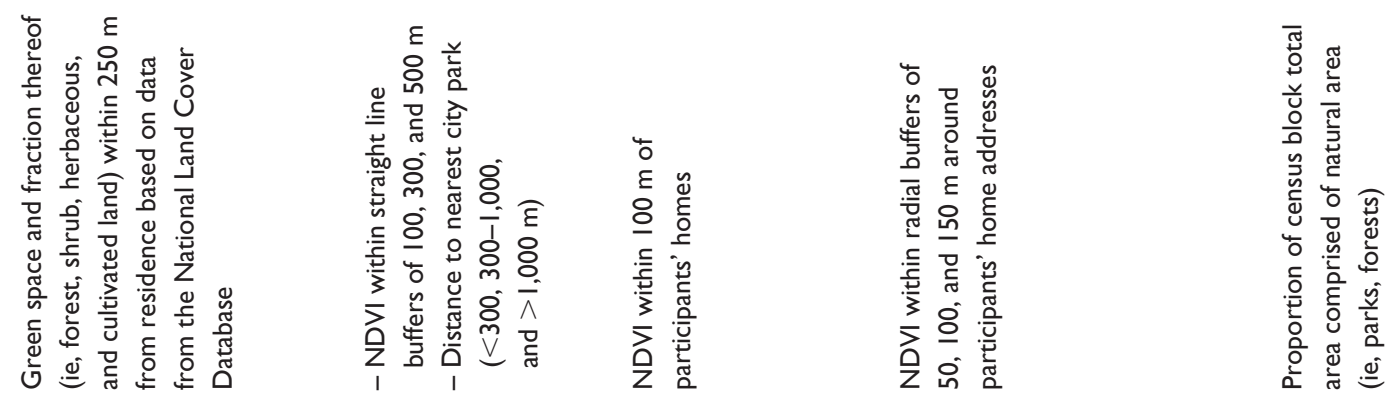

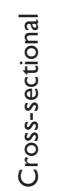

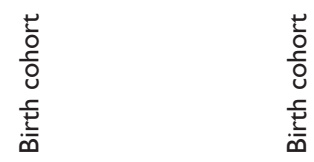

$\frac{1}{0}$
$\frac{0}{0}$
$\frac{0}{0}$
$\frac{0}{10}$<smiles>[B]=[B]</smiles>
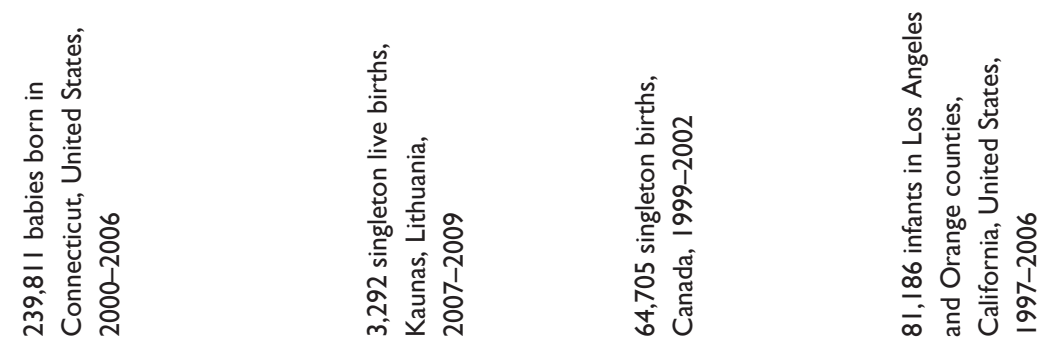

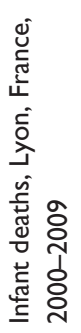
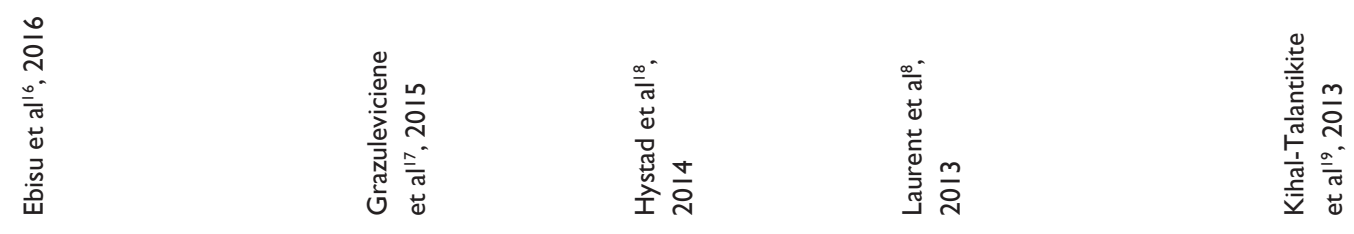


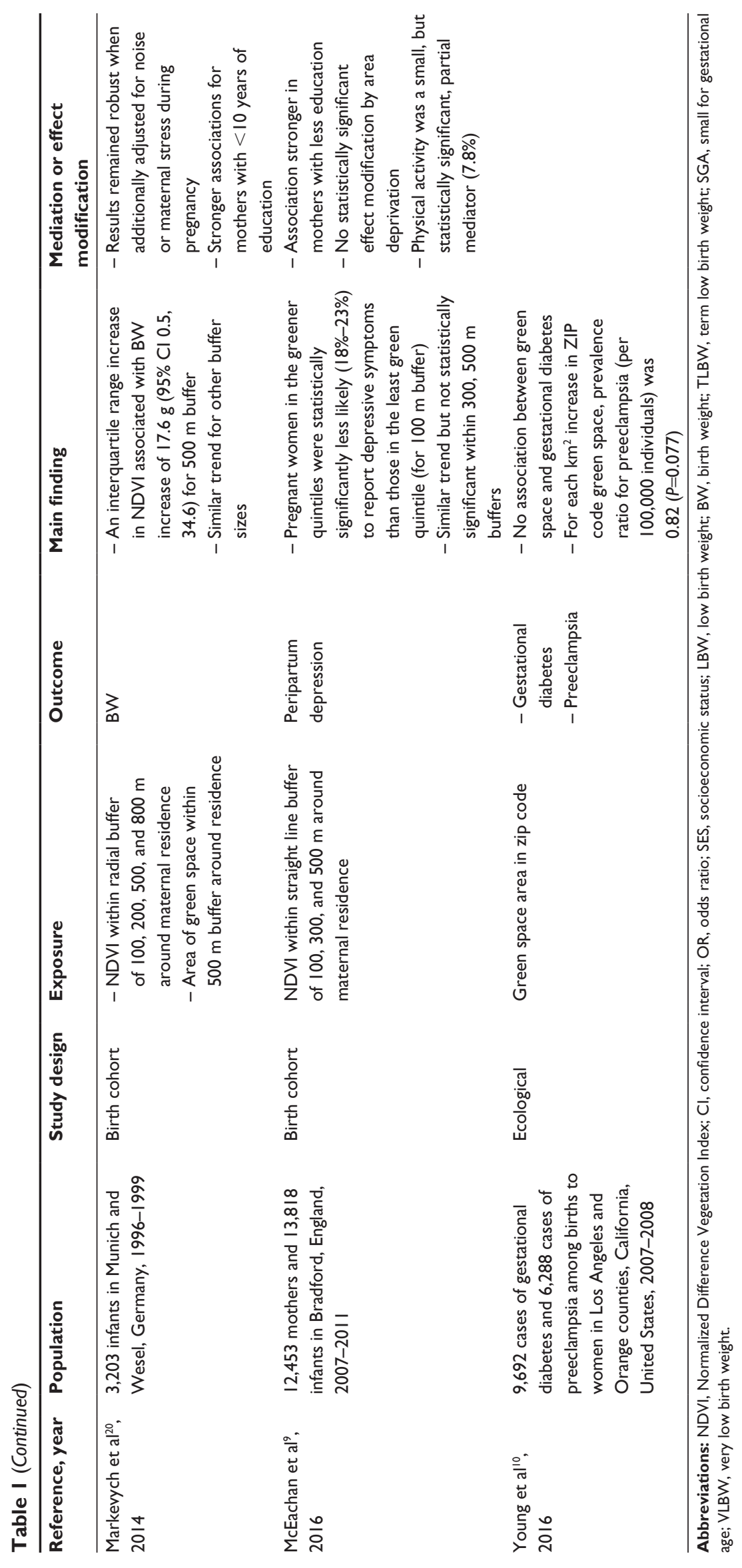




\section{Maternal health outcomes}

Relative to the literature on birth outcomes, greenness studies that considered maternal prenatal or peripartum health were few. Only three papers identified by the search discussed maternal health outcomes. ${ }^{8-10}$ These studies examined associations between greenness and gestational diabetes, preeclampsia, and peripartum depression. There appeared to be no association between greenness and gestational diabetes, and only a suggestive association, if any, between amount of greenness and preeclampsia in these studies. There did appear, however, to be a positive association between increasing greenness and odds of maternal depression in one study.

\section{Gestational diabetes}

Among women in Los Angeles and Orange counties, California, who gave birth in 2007 or 2008 , there was no statistically significant relationship between the amount of green space area in a ZIP code and the prevalence of gestational diabetes, in either unadjusted or adjusted models; however, this analysis did not adjust for maternal health and lifestyle factors such as diet, smoking, and alcohol use. ${ }^{10}$

\section{Preeclampsia}

Among women in Los Angeles and Orange counties, California, who gave birth in 2007 or 2008, there was no statistically significant relationship between the amount of green space area in a ZIP code and the prevalence of preeclampsia, in either unadjusted or adjusted models. However, there was a suggestive protective effect of the amount of green space on the prevalence of preeclampsia; for each $\mathrm{km}^{2}$ increase in green space in a ZIP code, the prevalence ratio for preeclampsia (per 100,000 individuals) decreased by $18 \%$ $(P=0.077)$. This ecological analysis was unable to adjust for maternal health and lifestyle factors such as diet, smoking, and alcohol use. ${ }^{10}$ Laurent et al considered neonatal records from four hospitals in Los Angeles and Orange counties between 1997 and 2006 in their retrospective cohort study on greenness and preeclampsia. ${ }^{8}$ In buffers of 50,100 , or $500 \mathrm{~m}$ around participants' home, there was no association between an interquartile range increase in NDVI and the risk of preeclampsia (odds ratio [OR] for $100 \mathrm{~m}$ buffer: $0.97,95 \%$ confidence interval [CI] 0.93, 1.01).

\section{Peripartum depression}

In a 2015 study, McEachan et al studied a birth cohort in Bradford, England, of 12,453 mothers between 2007 and 2011 and examined the association between greenness around participants' homes and peripartum depression..$^{9}$ They analyzed the relationship between greenness in radii of varying sizes around the residence and severe depression symptoms based on the General Health Questionnaire. They found that within buffers of 100,300, and $500 \mathrm{~m}$, those in the greenest quintiles had a statistically significantly reduced risk of peripartum depression compared to those in the least green quintile. The authors were able to adjust for a range of behavioral and socioeconomic factors including cohabitation status, financial struggles, smoking, alcohol use, and physical activity. When they replicated this analysis instead using the presence of a major green space $\left(5,000 \mathrm{~m}^{2}\right)$ within $300 \mathrm{~m}$ of the home address as the exposure of interest, they again found that the odds for peripartum depression for those in proximity to a major green space were reduced (OR 0.87, 95\% CI 0.770, 0.995).

\section{Infant health outcomes}

Twelve studies considered the relationship between greenness and infant health outcomes, including gestation length, preterm birth, continuous birth weight, LBW, and SGA. ${ }^{7,8,11-20}$ Outcome data were gathered from birth certificates or clinical examinations. Greenness was generally associated with higher birth weight and lower odds of LBW, but associations between greenness and gestation length or preterm birth were mostly null.

\section{Birth weight}

Birth weight was the most commonly investigated neonatal outcome in studies of greenness. There was a consistent trend toward a positive relationship between greenness and birth weight across multiple countries, although the magnitude of effect sizes was relatively small, suggesting a weak association.

Most studies of birth weight accounted for gestation length, most commonly by adjusting for it in regression models. In a study of infants born in Canada, Hystad et al observed birth weight was $20.4 \mathrm{~g}$ higher for each 0.1 unit increase in NDVI in a $100 \mathrm{~m}$ buffer around the postal code centroid. ${ }^{18}$ Exposure was assigned using mother's addresses across the full duration of pregnancy, and the authors adjusted for maternal socioeconomic status and smoking, which may be important confounders in the relationship between greenness and birth weight. Studies in the United Kingdom ${ }^{14}$ and Spain ${ }^{13}$ found similar estimates of higher birth weight in greener areas, between 16 and $44 \mathrm{~g}$ per interquartile range increase in NDVI depending on the buffer size. These studies were able to adjust for a variety of potential confounders including maternal behaviors such as smoking and alcohol use. However, they relied on a single measure of exposure based 
on address at time of delivery. Studies in the United States, ${ }^{8}$ Israel, ${ }^{11}$ and Germany ${ }^{20}$ also reported statistically significantly higher birth weights in greener areas, with effect sizes ranging from 3.2 to $19.2 \mathrm{~g}$ heavier for an interquartile range increase in greenness. Not all studies that assessed the relationship between greenness and birth weight observed associations: studies of babies born in Lithuania, ${ }^{17}$ Spain, ${ }^{12}$ and the United States $^{7}$ did not find any statistically significant relationship. The study conducted in Lithuania observed $19.8 \mathrm{~g}$ lower birth weight per $10 \%$ increase in greenness $(95 \%$ CI -67.6, 28.1), whereas the studies in Spain and the United States observed positive but nonsignificant associations between greenness and birth weight (2-42 g higher birth weight depending on the exposure metric).

Some studies considered the dichotomous outcomes of LBW ( $<2,500 \mathrm{~g})$, very low birth weight (VLBW, $<1,500 \mathrm{~g})$, or SGA (10th percentile or less in weight compared to babies of the same gestational age). Studies that considered both continuous and dichotomous birth weight outcomes generally arrived at results in the same direction. Consistent with their finding for continuous birth weight, Agay-Shay et al observed a statistically significant $16 \%$ decrease in the odds of LBW for an interquartile range increase in greenness; results for VLBW were in the same direction but not statistically significant. ${ }^{11}$ Grazuleviciene et al did not find any statistically significant association between greenness and LBW, with an OR of 0.97 for an interquartile range increase in all greenness measures reported. ${ }^{17}$ Overall results for the dichotomous outcome of SGA were similar to those for LBW, with studies reporting between $3 \%$ and $27 \%$ lower odds of SGA for an interquartile range increase in greenness.

Although most of the studies considered surrounding greenness, as measured by NDVI, as the exposure of interest, some studies did consider whether proximity to parks, forests, or other green spaces was associated with birth weight. ${ }^{15-17,20}$ Results for these measures were mixed: among babies born in the United States, a greater proportion of park land near the residence was associated with 7.6\% lower risk of LBW, ${ }^{16}$ and a $10 \%$ increase in tree cover was associated with a $1 \%$ reduction in the odds of SGA. ${ }^{15}$ Conversely, a study in Germany found no relationships between distance to parks or forests and birth weight, ${ }^{20}$ and one in Lithuania observed statistically significantly increased odds of LBW only for those who lived both far from a park and in a low NDVI area (OR 2.23, 95\% CI 1.20, 4.15). ${ }^{17}$

\section{Gestation length}

Multiple studies considered outcomes related to gestation length, either as a continuous measure in weeks or dichotomized. Results were mixed, with most studies finding no statistically significant relationship between greenness and gestation length or preterm birth. In addition, although gestation length would seem to provide a pathway to birth weight, it did not appear to be the case that positive birth weight outcomes were driven by gestation length.

The most common dichotomized outcome was preterm ( $<37$ weeks) versus term birth, but some studies considered very preterm $(<30$ weeks) and moderately preterm (30-36 weeks) birth separately. Casey et al found an inverse relationship between greenness and preterm birth in babies born in Pennsylvania cities (OR 0.78 comparing the two greenest tertiles to the least green, 95\% CI $0.61,0.99)$; in townships and boroughs, areas of lower population density, there were no statistically significant associations. This study assigned exposure based on the NDVI values for the seasons of gestation rather than relying on a single time point. The authors were also able to adjust for a variety of behavioral confounders (including smoking, body mass index, and antibiotic use) and environmental confounders (including well water, neighborhood walkability, and distance to road). ${ }^{7}$ In a Vancouver cohort, Hystad et al observed statistically significantly lower odds of moderately preterm birth (OR 0.95, 95\% CI $0.91,0.99)$ in greener areas, but no significant decrease in the odds of very preterm birth (OR $0.91,95 \%$ CI 0.77 , 1.07). ${ }^{18}$ Other studies conducted in Spain, ${ }^{12,13}$ the United States, ${ }^{15}$ Israel, ${ }^{11}$ and Lithuania ${ }^{17}$ found no association between greenness and preterm birth or continuous gestational age. Effect sizes in these null studies were small and inconsistent in direction, with ORs for preterm between 0.98 and 1.07, and beta coefficients for gestational age between 0 and 0.3 days.

\section{Other outcomes}

One ecological study considered the relationship between the proportion of census block total area that is natural area and a geographic cluster of infant mortality. The presence of natural areas explained only a small fraction of this cluster of infant mortality. ${ }^{19}$ This study was limited by the ecological design and the analytic approach, which focused only on one cluster of infant mortality.

Very few studies to date have assessed other anthropometric or developmental outcomes. One study assessed the relationship between Apgar score and greenness during pregnancy and observed no associations. ${ }^{7}$ Head circumference was also considered in one study; the authors reported $1.7 \mathrm{~mm}$ larger head circumference for an interquartile range increase in NDVI. ${ }^{13}$ 


\section{Mediation analyses}

Although some studies presented results for models both adjusted and unadjusted for certain key variables, only one study conducted a formal mediation analysis. McEachan et al found that within the 100,300, and $500 \mathrm{~m}$ buffers in which NDVI was assessed, physical activity was a partial but statistically significant mediator (between $5.6 \%$ and $7.8 \%$ ) of the effect of greenness on peripartum depression. ${ }^{9}$

Other studies stopped short of a mediation analysis but compared effect estimates including or excluding certain other key variables. Laurent et al presented results including estimates of traffic density and air pollutant concentrations; statistically significant associations between greenness and birth weight persisted in the $50 \mathrm{~m}$ buffer after adjustment for air pollutants, but not in the $100 \mathrm{~m}$ buffer. ${ }^{8}$ The statistically significant association between an interquartile range increase in greenness within a $150 \mathrm{~m}$ buffer became nonsignificant after adjustment for particulate matter (2.5 microns) or traffic density. However, the authors did find statistically significantly decreased risks within the 50 and $100 \mathrm{~m}$ buffers only after adjusting for NOx or CO. ${ }^{8}$ In their study, Hystad et al found a statistically significant positive relationship between NDVI and birth weight, and lower odds of moderately preterm birth in greener areas. ${ }^{18}$ These associations persisted after adjustment for other spatially correlated features such as air pollution exposure, noise exposure, walkability, and park distance; adjustment for individual and area socioeconomic status, however, attenuated the associations. Overall, these findings suggest that the relationship between greenness, pollution, and birth weight is complex and the nature of the associations may not be the same for all pollutants. The fact that associations between greenness and birth outcomes persisted after adjustment for key pollutants suggests that buffering of other harmful environmental exposures does not fully account for the observed associations.

\section{Effect modification analyses}

Many studies considered effect measure modification, in particular whether the relationship between greenness and birth outcomes differed across household and neighborhood measures of socioeconomic status. Results were mixed, but multiple studies found stronger associations between greenness and birth outcomes in participants or neighborhoods of lower socioeconomic status. ${ }^{12-14,16,20}$ These findings suggest that the potential benefits of greenness on health may be strongest in more deprived areas.

Several studies observed that the relationship between greenness and birth outcomes was stronger in mothers with lower levels of education or lower household income, ${ }^{12-14,16,20}$ suggesting that individual education and income may be important moderators of the relationship between greenness and health. These findings concur with studies of greenness and other health outcomes, which have also found stronger relationships in individuals of lower socioeconomic status. ${ }^{2}$ Effect modification by individual race or ethnicity was less clear: Dadvand et al observed a relationship between greenness and birth weight in white but not Pakistani residents of Great Britain, ${ }^{14}$ whereas in studies of residents of the United States ${ }^{16}$ and Canada, ${ }^{18}$ there were no differences in associations across racial or ethnic groups.

A total of seven studies assessed the presence of effect modification by some measures of neighborhood socioeconomic status such as a community deprivation score or median household income. Although some of these studies saw a trend toward stronger relationships in areas of lower socioeconomic status, ${ }^{11,14,18}$ the majority observed no statistically significant evidence of effect modification by area socioeconomic status. ${ }^{7,9,10,19}$

One study also assessed potential effect modification by maternal physical activity. For example, McEachan et al tested whether the relationship between NDVI and peripartum depression differed by physical activity level, and found that the beneficial effect of greenness was stronger in those who were physically active, only in the $300 \mathrm{~m}$ buffer. ${ }^{9}$ There was no evidence of effect modification by physical activity in their analysis concerning proximity to a major green space.

\section{Discussion}

The relationship between greenness and maternal health and birth outcomes is an emerging area of research. Studies across several countries have observed a relationship between greenness and birth weight, with babies born in greener areas likely to be heavier. Results for gestation length were less consistent, with the majority of studies finding no associations between greenness and gestation length. The majority also found no association with risk of preterm birth, although some studies did find lower odds of preterm birth among babies born in greener areas. Only three studies considered maternal outcomes, such as preeclampsia or gestational diabetes. The two studies reviewed on this topic generally did not find statistically significant associations; however, one study identified a relationship between higher greenness and reduced odds of preeclampsia. One study also found that increased greenness was statistically significantly associated with lower odds of peripartum depression. This finding was consistent with a larger body of literature suggesting that greenness may be associated with lower risk of depression 
and distress in diverse populations. ${ }^{2}$ Overall, the results of existing studies suggest that greenness may be positively associated with desirable neonatal outcomes such as birth weight and gestation length and that greenness may be associated with lower odds of detrimental maternal outcomes, particularly peripartum depression, though only one study considered this association.

In studies that were able to evaluate effect modification, there was suggestive evidence that the relationship between greenness and health may be stronger in people of lower socioeconomic status. Only one study evaluated effect modification by maternal behaviors and observed the relationship between greenness and health was stronger in physically active mothers. Many studies had relatively few cases and therefore limited power to assess effect modification, so findings should be interpreted cautiously and further research is needed. Few studies addressed mediation.

Most studies relied on exposure characterized at a single point in time (usually based on residence at the time of birth). This approach may not represent exposure through the duration of pregnancy, particularly if mothers moved residences. Only one study considered seasonal variations in greenness in their exposure characterization. ${ }^{7}$ Given relatively fine temporal resolution at which vegetation indices such as NDVI are available, the impact of seasonal variations in greenness on pregnancy outcomes represents an important and accessible area for future research. A more nuanced exposure characterization would also allow for the exploration of critical periods of exposure during pregnancy.

The relationship between socioeconomic status, race/ethnicity, and greenness exposure is a multidimensional one. In the US, those with greater socioeconomic status, more likely to be white, may also have greater greenness exposure in cities, where such real estate is more expensive. ${ }^{21,22}$ However, in suburban or rural settings, there may be fewer associations between socioeconomic status, race/ethnicity, and greenness - or they may run the other way. ${ }^{23}$ Even within the US, these relationships are likely to vary by geography. In cohorts outside the US, where most of the studies reviewed were conducted, the relationships between class, race/ethnicity, and greenness manifest differently. In general, the studies reviewed, across all outcomes, were able to adjust for socioeconomic status and race/ethnicity, for example, by including terms for maternal level of education or material deprivation in adjusted models. Given the complexity of the concepts and the scant availability of proxies in some scenarios, adjustment for socioeconomic status may not be complete. However, it is unlikely - given the nature of the relationship between socioeconomic status and greenness, and the ability of most researchers to account for socioeconomic status in some way - that socioeconomic status explains all or even most of the associations observed between greenness and health.

The relationship between the physical environment and health is complex, ${ }^{24}$ and greenness is just one component that may influence maternal health and birth outcomes. Very few studies considered other relevant characteristics of the physical environment such as walkability, perceived safety, access to destinations, or land use, either as covariates or separate exposures. ${ }^{7,16,18}$ Future research should investigate not only the relationship between greenness and health but also the interrelationships between nature and other components of the physical environment that may interact to influence health.

Although research in this area has begun to assemble an evidence base on the effects of greenness on maternal health and birth outcomes, more studies are needed to validate these results, explore other outcomes, and identify mechanisms. Although particular birth outcomes like preterm birth and birth weight have received some scholarly attention, more developmental-type assessments (eg, Apgar score) could help fill out the picture for the relationship between greenness and infant health. Similarly, despite small inroads, little is still known about the relationship between greenness and maternal health outcomes. There may be particular unexplored links between greenness and maternal mental health.

The methods these studies employ could also be improved in several key respects. Most studies in this realm were cross-sectional, and even those that occurred in prospective cohorts often only assessed greenness exposure around birth. Therefore, these studies may misclassify exposure during pregnancy and obscure the temporality of exposure and outcome. Relatedly, studies on greenness and birth outcomes should focus on critical periods; because pregnancy is a specific and temporary state, prenatal greenness exposure should consider key windows and seasonal fluctuations.

In a research area where the causal pathways remain unclear, few studies employed mediation analyses. Although some considered air pollution and other environmental features as potential mediators, physical activity, social interaction, a host of other factors may play mediating roles that could be elucidated.

Finally, as with any birth cohort study, the ones summarized in this review may be subject to selection bias. If the exposure is associated with viability, those births eligible for inclusion in a birth cohort are limited to those unaffected by the exposure during gestation - a subject must have survived 
to the time of birth to be included in the study. The extent of this bias would be dependent on how likely greenness may be to influence viability. In this context, selection bias introduced via this mechanism is likely to be small, but future studies that rely on pregnancy cohorts, where mothers are followed from conception or prior to conception, rather than birth cohorts, would help address this issue.

There is growing evidence that greenness may be associated with improved birth and pregnancy outcomes. Further research is needed to understand the relevant mechanisms, and future studies should therefore consider mediation analysis, with particular attention to whether frequently studied pathways such as increased physical activity and social interaction or lower exposure to harmful environmental exposures such as air pollution and noise explain the relationships between greenness and birth outcomes. Given the evidence indicating that positive relationships between greenness and birth outcomes may be stronger in those of lower socioeconomic status, future research should continue to explore effect modification by individual and area socioeconomic status. Overall, this review indicates a potential positive relationship between greenness exposure and a number of birth outcomes in several studies, and underlines the need for more research replicating these findings and exploring their implications in more detail.

\section{Acknowledgments}

The authors would like to thank Maggie Mittleman for her help identifying the articles for this review. This study was funded by NIH T32 ES 007069, K99 CA201542, P30 ES000002 (Hart), and American Heart Association Scientist Development Grant \#13SDG14580030.

\section{Disclosure}

The authors report no conflicts of interest in this work.

\section{References}

1. Bedimo-Rung AL, Mowen AJ, Cohen DA. The significance of parks to physical activity and public health: a conceptual model. Am J Prev Med. 2005;28(2 Suppl 2):159-168.

2. James P, Banay RF, Hart JE, Laden F. A review of the health benefits of greenness. Curr Epidemiol Rep. 2015;2(2):131-142.

3. Berkman L, Kawachi I. Social epidemiology. New York, NY, USA: Oxford University Press; 2000.
4. Nowak DJ, Crane DE, Stevens JC. Air pollution removal by urban trees and shrubs in the United States. Urban For Urban Green. 2006;4: $115-123$.

5. Gidlöf-Gunnarsson A, Öhrström E. Noise and well-being in urban residential environments: the potential role of perceived availability to nearby green areas. Landsc Urban Plann. 2007;83:115-126.

6. Lafortezza R, Carrus G, Sanesi G, Davies C. Benefits and well-being perceived by people visiting green spaces in periods of heat stress. Urban For Urban Green. 2009;8(2):97-108.

7. Casey JA, James P, Rudolph KE, Wu CD, Schwartz BS. Greenness and birth outcomes in a range of Pennsylvania communities. Int J Environ Res Public Health. 2016;13(3):E311.

8. Laurent O, Wu J, Li L, Milesi C. Green spaces and pregnancy outcomes in Southern California. Health Place. 2013;24:190-195.

9. McEachan RR, Prady SL, Smith G, et al. The association between green space and depressive symptoms in pregnant women: moderating roles of socioeconomic status and physical activity. J Epidemiol Commun Health. 2016;70(3):253-259.

10. Young C, Laurent O, Chung JH, Wu J. Geographic distribution of healthy resources and adverse pregnancy outcomes. Matern Child Health J. 2016; 20(8):1673-1679.

11. Agay-Shay K, Peled A, Crespo AV, et al. Green spaces and adverse pregnancy outcomes. Occup Environ Med. 2014;71(8):562-569.

12. Dadvand P, de Nazelle A, Figueras F, et al. Green space, health inequality and pregnancy. Environ Int. 2012;40:110-115.

13. Dadvand P, Sunyer J, Basagana X, et al. Surrounding greenness and pregnancy outcomes in four Spanish birth cohorts. Environ Health Perspect. 2012;120(10):1481-1487.

14. Dadvand P, Wright J, Martinez D, et al. Inequality, green spaces, and pregnant women: roles of ethnicity and individual and neighbourhood socioeconomic status. Environ Int. 2014;71:101-108.

15. Donovan GH, Michael YL, Butry DT, Sullivan AD, Chase JM. Urban trees and the risk of poor birth outcomes. Health Place. 2011;17(1): 390-393.

16. Ebisu K, Holford TR, Bell ML. Association between greenness, urbanicity, and birth weight. Sci Total Environ. 2016;542(Pt A):750-756.

17. Grazuleviciene R, Danileviciute A, Dedele A, et al. Surrounding greenness, proximity to city parks and pregnancy outcomes in Kaunas cohort study. Int J Hyg Environ Health. 2015;218(3):358-365.

18. Hystad P, Davies HW, Frank L, et al. Residential greenness and birth outcomes: evaluating the influence of spatially correlated builtenvironment factors. Environ Health Perspect. 2014;122(10):1095-1102.

19. Kihal-Talantikite W, Padilla CM, Lalloue B, Gelormini M, Zmirou-Navier D, Deguen S. Green space, social inequalities and neonatal mortality in France. BMC Pregnancy Childbirth. 2013;13:191.

20. Markevych I, Fuertes E, Tiesler CM, et al. Surrounding greenness and birth weight: results from the GINIplus and LISAplus birth cohorts in Munich. Health Place. 2014;26:39-46.

21. Conway D, Li C, Wolch J, Kahle C, Jerrett M. A spatial autocorrelation approach for examining the effects of urban greenspace on residential property values. J Real Estate Finan Econ. 2010;41(2):150-169.

22. Polyakov M, Pannell DJ, Pandit R, Tapsuwan S, Park G. Capitalized amenity value of native vegetation in a multifunctional rural landscape. Am J Agric Econ. 2015;97(1):299-314.

23. Wen M, Zhang X, Harris C, Holt J, Croft J. Spatial disparities in the distribution of parks and green spaces in the USA. Ann Behav Med. 2013; 45(Suppl 1):18-27.

24. Diez Roux AV, Mair C. Neighborhoods and health: neighborhoods and health. Ann N Y Acad Sci. 2010;1186(1):125-145. 


\section{Publish your work in this journal}

The International Journal of Women's Health is an international, peerreviewed open-access journal publishing original research, reports, editorials, reviews and commentaries on all aspects of women's healthcare including gynecology, obstetrics, and breast cancer. The Visit http://www.dovepress.com/testimonials.php to read real quotes from published authors.

manuscript management system is completely online and includes

Submit your manuscript here: http://www.dovepress.com/international-journal-of-womens-health-journal 